Manuelle Medizin 2009 · 47:233-234

DOI 10.1007/s00337-009-0700-3

Online publiziert: 2 . August 2009

๑) Springer Medizin Verlag 2009

L. Beyer ${ }^{1} \cdot$ R. Kayser ${ }^{2}$

${ }^{1}$ Ärztehaus Mitte, Jena

${ }^{2}$ Klinik und Poliklinik für Orthopädie und Orthopädische Chirurgie, Universitätsklinikum Greifswald

\title{
Die manuelle Medizin ist ein unverzichtbares Werkzeug des Arztes
}

Liebe Leserin, lieber Leser!

Angesichts reformierter Reformen von Reformen und finanziellen Beschneidungen ist man einen kurzen Moment versucht, dem Editorial die Überschrift „Yes we can“ zu geben. Aber so schlimm ist es wohl nicht, dass wir uns als Selbsthilfegruppe finden müssen.

Wir können darauf vertrauen:

Die manuelle Medizin ist eine alte Heilkunst.

Wie kaum in Lehrbüchern anderer Fächer wird in der manuellen Medizin und der Osteopathie fast immer auf schillernde Bilder der frühen Entwicklung unseres Faches eingegangen. Medizinhistorisch lassen sich in allen Jahrhunderten Hinweise auf das Untersuchen und Behandeln mit der Hand finden. Wissenschaftliche Gesellschaften, die sich intensiv der manuellen Medizin widmen, gründeten sich aber erst im letzten Jahrhundert.

Unter diesem wissenschaftlichen Aspekt, der systematischen Analyse der Untersuchungen und den zugrunde liegenden Mechanismen der Testergebnisse, der Strukturierung der Chirotherapie und Aufklärung ihrer Wirkungsmechanismen kann man auch sagen:

Die manuelle Medizin ist ein junges Fachgebiet.

In den letzten Jahren hat die manuelle Medizin eine stürmische Entwicklung in
Europa erlebt. Es haben sich Ärztegesellschaften formiert, die sich der Verbreitung der Methoden der manuellen Medizin widmen. Hinzu kommen zahlreiche Schulen, die die Techniken an Ärzte und Physiotherapeuten weitervermitteln, manuelle Techniken unter dem Namen der manuellen Medizin und ihrer Teilgebiete: der Chirotherapie, der manuellen Therapie, der Osteopathie und der Chiropraktik. In einem Leserbrief wird sogar die Frage gestellt, ob nicht $\mathrm{zu}$ viele Manualtherapeuten ausgebildet werden? In Kontakten mit Journalisten, bei Interviews und Fernsehsendungen kommt aber sehr oft die Frage, was ist denn eigentlich manuelle Medizin? Bedeutet dies für uns:

Die manuelle Medizin ist ein kleines Fachgebiet?

Zunehmend interessieren sich klinische Fachgebiete für inzwischen aufgedeckte Zusammenhänge zwischen einzelnen Symptomen von Dysfunktionen an verschiedenen Teilen des Bewegungssystems und klinischen Befunden ihres eigenen Faches. Besonders deutlich entwickelt sich in den letzten Jahren eine Zusammenarbeit mit Kinderärzten und Zahnmedizinern. In diesem Sinne kann man vielleicht schon sagen:

Die manuelle Medizin ist ein breites Fachgebiet.

Ihre Nutzung in den klinischen Fachgebieten bringt aber Verpflichtungen für die manuelle Medizin. Sie muss ihre Methoden gewichten und bewerten. Sie versucht, sich Kriterien einer evidenzbasierten Medizin zu stellen, sie beginnt, eigene Forschung zu betreiben. Wenn man ganz mutig ist, kann man bis zu über 50 Zeitschriften zusammentragen, die für die manuelle Medizin relevante wissenschaftliche Ergebnisse darstellen. Die Mehrheit wissenschaftlicher Studien kommt aus den USA. Sie wird von den gut durchorganisierten Verbänden der Chiropraktoren und Osteopathen erbracht. Aber auch in Europa beginnt sich etwas zu regen. So wurde eine Europäische Wissenschaftliche Gesellschaft für Manuelle Medizin gegründet (ESOMM). In Prag trafen sich im Mai Wissenschaftler unter dem Namen „Internationale Akademie für muskuloskeletale/manuelle Medizin“ (http:// www.iammm.net). Im Schweizer Horn am Bodensee diskutierten auf Einladung der ESOMM Vertreter der täglich angewandten manuellen Medizin mit Grundlagenwissenschaftlern. Und vielleicht besonders hervorzuheben ist: Es gibt einen kleinen Andrang nach den Seminaren der Forschungsberatungsstelle der DGMM.

Fast unbeobachtet beginnt ein Prozess einer Akademisierung der manuellen Medizin. Erste fakultative und obligatorische Vorlesungen sind in das Medizinstudium eingezogen. Die Verbände der Physiotherapeuten streben einen Master in manu- 
eller Therapie an. Bereits an Studenten wird vermittelt: Ursachen von Schmerzen kann man manuell untersuchen und kostensparend manuell behandeln. In diesem Sinne begreifen sie frühzeitig:

Die manuelle Medizin ist Schmerztherapie.

Meist kommen diese Schmerzen aus dem Bewegungssystem. Dadurch ist die manuelle Medizin eng mit muskulären Beschwerden und der nervalen Steuerung der Motorik verknüpft, sodass nicht nur die FIMM ihr MM als „muskuloskeletale/ manuelle Medizin“ versteht. Bisher hat wohl niemand so intensiv die bekannten physiologischen Prozesse der menschlichen Motorik, des menschlichen Bewegungssystems mit der manuellen Medizin verbunden wie Prof. Karel Lewit in seinem allen bekannten Buch Manuelle Medizin, dessen momentane 8. Auflage noch nicht die letzte sein wird. Lewit arbeitet ganz stark die funktionelle Seite der erhobenen Befunde und Therapieansätze heraus, sodass er formuliert:

Die manuelle Medizin ist eine muskuloskeletale Medizin

und (aufgrund vieler ihrer Wirkmechanismen)

die manuelle Medizin ist eine Reflextherapie.1

Fasst man die oben dargestellte Entwicklung zusammen, so wird die Stellung der manuellen Medizin in der Schulmedizin deutlich. Im Vergleich zur apparativen Untersuchung ist die manuelle Untersuchung aber eine unverzichtbare Alternative, genauso wie die manuelle Behandlung eine Alternative oder ergänzende Alternative zur medikamentösen Behandlung bei vielen funktionellen Beschwerden nicht nur, aber insbesondere, am Bewegungssystem ist. So gesehen ist es kein Widerspruch, wenn Vertreter der Naturheilkunde oder alternativer medizinischer Richtungen sich auch der Untersuchung und Behandlung mit der Hand bedienen.

Die Kunst der manuellen Untersuchung und die Kunst der manuellen Therapie sind und bleiben ein allgemeines und in vielen Fällen auch notwendiges spezielles „Handwerkszeug“ des Arztes, dessen Einsatz er gezielt erwerben muss,

1 Lewit K (1997) Manuelle Medizin. Barth, Heidelberg, 495 ff. um es gezielt einzusetzen, wie es wohl die Mehrheit unserer Leserinnen und Leser in der täglichen Praxis können - Yes, wir tun's.

Ihnen allen viel Erfolg dabei!

Mit freundlichen Grüßen
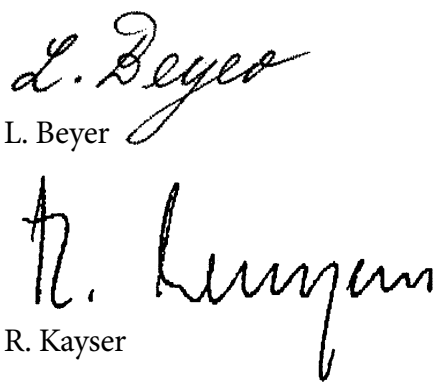

\section{Korrespondenzadresse}

Prof. Dr. L. Beyer

Ärztehaus Mitte

Westbahnhofstraße 2, 07745 Jena

lobeyer@t-online.de

\section{PD Dr. R. Kayser}

Klinik und Poliklinik für Orthopädie

und Orthopädische Chirurgie,

Universitätsklinikum Greifswald

Ferdinand-Sauerbruch-Straße, 17475 Greifswald

ralph.kayser@uni-greifswald.de

\section{Schmerzforum der DIVS}

Ein Jahr Fibromyalgie-Leitlinie sind wir weiter?

Wahrscheinlich zwischen 5 und $10 \%$ vor allem der älteren Deutschen leiden unter chronischen Schmerzen in mehreren Körperregionen ("chronic widespread pain", CWP). Das omyalgiesyndrom(FMS) ist ein möglicher Endpunkt eines CWP und betrifft bis zu 1 Mill. Menschen, überwiegend Frauen. Das Beschwerdebild ist mit hohen Krankheitskosten verbunden. Existenz und Klassifikation des FMS (rheumatologische Erkrankung? Psychische Störung? Schmerzkrankheit?) und seine Therapie werden zwischen den medizinischen Fachgebieten, den Behandlern und den Patienten kontrovers diskutiert.

Die DIVS erkannte 2006 als dringliche Aufgabe, das vorhandene klinische und empirische Wissen über das FMS zusammenzufassen, um so Betroffenen und Behandlern die bestmöglichen Handlungsempfehlungen geben zu können. Anfang 2008 wurde die interdisziplinär erarbeitete S3-Leitlinie(LL) zu „Definition, Pathophysiologie, Diagnostik und Therapie des Fibromyalgiesyndroms" ins Netz gestellt und im Juni 2008 in "Der Schmerz" publiziert. Wo stehen wir mehr als 1 Jahr danach? Was wurde erreicht? Was hat sich verändert? In Zusammenarbeit mit dem Interdisziplinären Schmerztherapiezentrum (IST) Mainz wollen wir dies am 14. November 2009 in Mainz diskutieren. Hierzu möchten wir Sie ganz herzlich einladen. Wir freuen uns sehr, Sie im Hörsaal Chirurgie der Universitätsmedizin im herbstlichen Mainz begrüßen zu dürfen.

H. Laubenthal, Präsident

F. Birklein, Vizepräsident, Sprecher IST Mainz W. Häuser, Generalsekretär, LL-Kommission FMS

J. Nadstawek, Schatzmeister

M. Schiltenwolf, Vizepräsident

Veranstaltungsort:

Universitätsmedizin Mainz, Hörsaal Chirurgie, Langebeckstr. 1, 55131 Mainz

Auskunft:

wikonet GmbH, Tel. 0611/2048090

Anmeldung per Fax bis 7.11.:

0611/20480910 